TRANSACTIONS OF THE

AMERICAN MATHEMATICAL SOCIETY

Volume 351, Number 7, Pages 2675-2693

S $0002-9947(99) 02407-1$

Article electronically published on March 8, 1999

\title{
CH WITH NO OSTASZEWSKI SPACES
}

\author{
TODD EISWORTH AND JUDITH ROITMAN
}

\begin{abstract}
There are models of $\mathrm{CH}$ without Ostaszeswki spaces. If $X$ is locally compact and sub-Ostaszewski, there is a forcing $P_{X}$ which does not add reals and which forces " $X$ is not sub-Ostaszewski".
\end{abstract}

\section{INTRODUCTION}

Ostaszewski spaces, originally constructed under $\diamond$ to answer a question about perfect normality (see [4]), have since proved useful both as counterexamples and as paradigms in many areas of general topology, and thus they are of some interest in their own right. As is often the case, the reflection argument implicit in a $\diamond$ construction can be easily modified to a forcing construction under, e.g., the partial order that simultaneously adds uncountably many Cohen reals, and variations with stronger properties were constructed using these methods. But it was not known if reflection was needed - in particular, it was unknown if Ostaszewski spaces could be constructed from $\mathrm{CH}$ alone. The aim of this paper is to show that they cannot.

Theorem 1. There are models of $\mathrm{ZFC}+\mathrm{CH}$ in which there are no Ostaszewski spaces.

The consistency is relative to the consistency of ZFC; our results require no large cardinals. Along the way, we establish the following:

Theorem 2. Suppose $X$ is a locally compact sub-Ostaszewski space. Then there is a notion of forcing $P_{X}$ that adds no reals such that

$$
\Vdash_{P_{X}} \text { "X is not a sub-Ostaszewski space". }
$$

Our proof defines $P_{X}$ so that it adds an uncountable set, each of whose countable subsets has compact closure. To show that $P_{X}$ is proper and adds no reals, we need only the assumptions of Theorem 2. In order to show that such forcings can be iterated without adding reals, we use the full definition of Ostaszewski spaces.

\section{TOPOLOGICAL BACKGROUND}

We gather here several elementary results concerning sub-Ostaszewski spaces; all are folklore.

Received by the editors December 20, 1996 and, in revised form, November 23, 1997.

1991 Mathematics Subject Classification. 03E35, 03E50, 54A35.

Key words and phrases. Ostaszewski space, Continuum Hypothesis, iterated forcing.

Research was done while the first author was a temporary assistant professor at the University of Kansas. 
Definition 2.1. A Hausdorff space $X$ is a sub-Ostaszewski space if $X$ is uncountable and every closed set is either countable or co-countable. If in addition $X$ is regular, countably compact, and non-compact, we call $X$ an Ostaszewski space.

Proposition 2.2. A sub-Ostaszewski space has at most one point with no countable neighborhood.

Proof. This follows immediately because in a sub-Ostaszewski space uncountable open sets cannot be disjoint, and thus given two points, the fact that our space is Hausdorff tells us that at least one of the points has a countable neighborhood.

Proposition 2.3. A locally countable space is hereditarily separable if and only if it has no uncountable discrete subspace.

Proof. Clearly an uncountable discrete subspace is not separable, so this proves one direction. For the other direction, suppose $Y$ is an uncountable non-separable subspace of a locally countable space $X$. Then it is straightforward to construct a subset $Z=\left\{x_{\alpha}: \alpha<\omega_{1}\right\}$ of $Y$ and a sequence $\left\{U_{\alpha}: \alpha<\omega_{1}\right\}$ of countable open subsets of $X$ such that $x_{\alpha} \in U_{\alpha}$ and $U_{\alpha} \cap\left\{x_{\beta}: \beta<\alpha\right\}=\emptyset$. Then $Z$ is an uncountable discrete subspace of $X$.

Proposition 2.4. A sub-Ostaszewski space is hereditarily separable.

Proof. Let $X$ be a sub-Ostaszewski space. By removing a point if necessary, we can assume that $X$ is locally countable. If $Y$ is an uncountable discrete subspace of $X$ and we partition $Y$ into uncountable sets $Y_{0}$ and $Y_{1}$, then $\operatorname{cl} Y_{0} \cap Y_{1}=\emptyset$, so $\operatorname{cl} Y_{0}$ is uncountable but not co-countable, a contradiction.

Proposition 2.5. Locally countable regular spaces are 0-dimensional.

Proof. Given $X$ locally countable, let $U$ be a countable neighborhood of some point $x \in X$. Since we assume $X$ is regular, there is an open neighborhood $V$ of $x$ such that $\operatorname{cl} V \subseteq U$. If $U=\operatorname{cl} V$ we are done. Otherwise, we use the fact that countable spaces are completely regular to get a continuous map $f: \operatorname{cl} V \rightarrow[0,1]$ which sends $x$ to 0 and $\operatorname{cl} V \backslash V$ to 1 . Let $\hat{f}$ be a continuous extension of $f$ to $X$ that sends $X \backslash V$ to 1 . Since $\operatorname{ran} \hat{f}$ is countable, we can find an $r \in[0,1] \backslash \operatorname{ran} \hat{f}$, and then $\hat{f}^{-1}([0, r))$ is a clopen neighborhood of $x$ contained in $U$.

Proposition 2.6. Ostaszewski spaces are locally countable.

Proof. Let $X$ be an Ostaszewski space and suppose $x$ has no countable neighborhood. Then every open neighborhood of $x$ is co-countable, which by countable compactness means every neighborhood of $x$ is co-compact. Hence $X$ is compact.

Proposition 2.7. Locally countable 0-dimensional countably compact spaces are locally compact; hence Ostaszewski spaces are locally compact.

Proof. This is immediate because a countable clopen set in a countably compact space is compact.

Proposition 2.8. A locally countable locally compact space is scattered.

Proof. If $Y$ is a subset of such a space, we can pick some countable open set $U$ that meets $Y$ and has compact closure. By a theorem of Mazurciewicz and Sierpinski, countable compact spaces are homeomorphic to ordinals, so there is some isolated point $x$ in $U \cap Y$. 
Proposition 2.9. A locally countable scattered space of size $\omega_{1}$ is right-separated in type $\omega_{1}$, i.e., the space can be well-ordered in type $\omega_{1}$ so that initial segments are open.

Proof. Let $X=\left\{x_{\alpha}: \alpha<\omega_{1}\right\}$ be locally countable and scattered. We re-enumerate $X$ as $\left\{y_{\alpha}: \alpha<\omega_{1}\right\}$ where $y_{0}$ is isolated in $X$, and each $y_{\alpha}$ is $x_{\beta}$ where $\beta$ is the least such that $x_{\beta}$ is isolated in $X \backslash\left\{y_{\gamma}: \gamma<\alpha\right\}$.

Proposition 2.10. A sub-Ostaszewski space has size $\omega_{1}$.

Proof. Let $X$ be a sub-Ostaszewski space. If $|X| \geq \omega_{2}$, then by the free set lemma for countable sets, there is a collection $\mathcal{U}$ of countable open sets, $|\mathcal{U}|=\omega_{2}$, so that for $U \in \mathcal{U}, U \backslash \cup[\mathcal{U} \backslash\{U\}] \neq \emptyset$. But then $X$ has a discrete subspace of size $\omega_{2}$, contradicting Proposition 2.4.

From now on, we assume $X$ is a topological space with $\omega_{1}$ as an underlying set and initial segments open - the above propositions show that in order to destroy all Ostaszewski spaces, it is sufficient to consider only those spaces $X$ with this form. Our convention is that topological notions refer to the topology $\tau$ on $X$, while ordering notions (e.g. "cofinal", "initial segment") refer to the usual order on $\omega_{1}$.

We now introduce some definitions and facts that are needed for our proof.

Definition 2.11. We write $\delta=X_{\delta}$ if every open set meeting $X \backslash \delta$ contains a sequence cofinal in $\delta$.

This choice of notation comes from the notation for the Cantor-Bendixson decomposition of scattered spaces.

Proposition 2.12. Let $X$ be hereditarily separable and $N$ be a countable elementary submodel of $H(\lambda)$ with $X \in N$. If $\delta=N \cap \omega_{1}$, then $\delta=X_{\delta}$.

Proof. Let $U$ be open in $X$ with $U \backslash \delta \neq \emptyset$. For purposes of contradiction, assume that $U \cap \delta$ is bounded by some $\gamma<\delta$. Since $\gamma \in N$, we can find (using hereditary separability) a countable set $D \subseteq X \backslash \gamma$ in $N$ such that $\operatorname{cl} D=X \backslash \gamma$. In particular, $U \cap D$ cannot be empty, and this contradicts our choice of $\gamma$.

An immediate corollary of the above proposition is that the set $\left\{\delta: \delta=X_{\delta}\right\}$ is closed and unbounded in $\omega_{1}$ if $X$ is hereditarily separable.

Definition 2.13. We say a set $Y$ is cofinally convergent in $\delta$ if

1. $Y \subseteq \delta$ is unbounded in $\delta$,

2. $\operatorname{cl} Y$ is compact,

3. there is some point $\operatorname{Top}(Y)$ such that every increasing $\omega$-sequence in $Y$ cofinal in $\delta$ converges to $\operatorname{Top}(Y)$.

We note that if $Y$ is cofinally convergent in $\delta$, then $\operatorname{Top}(Y)$ is unique and $\operatorname{Top}(Y) \geq \delta$.

Proposition 2.14. Let $Y$ be cofinal in $\delta$ where $\delta=X_{\delta}$. If $X$ is locally compact and $Y$ is open with compact closure, then there is some open $Z \subseteq Y$ cofinally convergent in $\delta$.

Proof. Let $\theta \geq \delta$ be the minimum element of $\operatorname{cl} Y \backslash \delta$. If $U$ is a compact open neighborhood of $\theta$ with $\sup U=\theta$, then $Y \cap U$ is the desired set. 
Corollary 2.15. If $X$ is countably compact and locally compact, $Y \subseteq X$ and $Y$ is open and cofinal in $\delta=X_{\delta}$, then $Y$ has an open subset $Z$ cofinally convergent in $\delta$.

Proposition 2.16. If $Y$ is open and cofinally convergent in $\delta$ where $\delta=X_{\delta}$, then for all compact open sets $U$, either $Y \backslash U$ is open and bounded below $\delta$, or $Y \cap U$ is open and bounded below $\delta ; Y \backslash U$ is bounded below $\delta$ if and only if $\operatorname{Top}(Y) \in U$.

\section{Total PROPERNESS AND ITERATED FORCING}

We assume familiarity with Shelah's work on proper forcing as presented in [5], [6], or [3]; in particular, we assume the reader is used to working with countable elementary submodels of $H(\lambda)$ - the sets whose hereditary cardinality is less than $\lambda$ - where $\lambda$ is some large enough regular cardinal. We assume (without loss of generality) that all of the notions of forcing with which we work are separative, i.e., if $q$ is not an extension of $p$, then $p$ has an extension $r$ that is incompatible with $q$. Notice that if $P$ is separative and both $p$ and $q$ are in $P$, then $p \Vdash q \in \dot{G}_{P}$ if and only if $p$ extends $q$; this will be of use later.

Our conventions regarding iterated forcing are standard (see [3] for example). If $\mathbb{P}=\left\langle P_{\alpha}, \dot{Q}_{\alpha}: \alpha<\kappa\right\rangle$ is a countable support iteration, we adopt the convention that $P_{0}$ is the trivial one element partial order for notational convenience. We will write $\Vdash_{\alpha}$ instead of $\Vdash_{P_{\alpha}}$. We will also make use of standard facts concerning quotient forcing. For example, if $\alpha<\beta<\kappa$, then by $P_{\beta} / \dot{G}_{\alpha}$, we mean a $P_{\alpha}$ name such that

$$
\Vdash_{\alpha} P_{\beta} / \dot{G}_{\alpha}=\left\{p \in P_{\beta}: p\left\lceil\alpha \in \dot{G}_{\alpha}\right\}\right.
$$

where $\dot{G}_{\alpha}$ is the canonical $P_{\alpha}$ name for the generic set adjoined by $P_{\alpha}$. As is wellknown, forcing with $P_{\beta}$ is essentially the same as forcing with $P_{\alpha} * P_{\beta} / \dot{G}_{\alpha}$. We use, at some point, the fact that if $r \in P_{\alpha}, \dot{s}$ is a $P$-name forced by $r$ to lie in $P_{\beta} / \dot{G}_{P_{\alpha}}$, and there is a countable set of ordinals $S$ in $V$ so that $r$ forces that the support of $\dot{s}$ is a subset of $S$, then $r * \dot{s} \in P_{\alpha} * P_{\beta} / \dot{G}_{P_{\alpha}}$ can be canonically translated into condition $r^{+} \in P_{\beta}$ satisfying $r^{+}\lceil\alpha=r$. The reader seeking more details should consult Section 4 of [3].

We now turn to the study of proper notions of forcing that add no new reals to the ground model. The notation $\operatorname{Gen}(N, P)$ and $\operatorname{Gen}^{+}(N, P)$ has its origins in [5].

Definition 3.1. Let $P$ be a notion of forcing, let $N \prec H(\lambda)$ be countable with $P \in N$, and let $p \in N \cap P$. We define

1. $\operatorname{Gen}(N, P)=\{G \subseteq N \cap P: G$ is an $N$-generic filter on $N \cap P\}$;

2. $\operatorname{Gen}^{+}(N, P)=\{G \in \operatorname{Gen}(N, P): G$ has a lower bound in $P\}$;

3. $\operatorname{Gen}(N, P, p)=\{G \in \operatorname{Gen}(N, P): p \in G\}$;

4. $\operatorname{Gen}^{+}(N, P, p)=\operatorname{Gen}(N, P, p) \cap \operatorname{Gen}^{+}(N, P)$.

In the sequel, we will have occasion to speak of several objects that are generic in certain senses. In general, when we are speaking of a notion of forcing $P$, we let $\dot{G}$ be the canonical $P$-name for the generic subset of $P$. When we are speaking of elements of some $\operatorname{Gen}(N, P)$, we will use an overset bar to indicate that we are referring to an object that is generic only over the model $N$, and when the bar is lacking we are referring to honest-to-goodness generic subsets of $P$. Thus we will see phrases like "let $\bar{G} \in \operatorname{Gen}(N, P)$ " or "let $G \subseteq P$ be generic".

Definition 3.2. With $N$ and $P$ as in the previous definition, we say a condition $q \in P$ is totally $(N, P)$-generic if $q$ is a lower bound of some $\bar{G} \in \operatorname{Gen}(N, P)$. We say 
$P$ is totally proper if for any countable $N \prec H(\lambda)$ containing $P$ and any $p \in N \cap P$, there is a totally $(N, P)$-generic condition $q \leq p$.

We will invest a bit of time developing some of the basic properties of totally proper forcing. None of the proofs are very difficult.

Proposition 3.3. For a notion of forcing $P$, the following conditions are equivalent:

1. $P$ is totally proper.

2. $P$ is proper, and forcing with $P$ adds no new function from $\omega$ to $V$.

3. $P$ is proper, and forcing with $P$ adds no new reals.

Proof. (1) implies (2): Suppose $\dot{f}$ is a $P$-name for a function from $\omega$ to $V$, and let $p \in P$ be arbitrary. We find a $q \leq p$ and $f \in V$ such that

$$
q \Vdash \dot{f}=f .
$$

Choose a countable $N \prec H(\lambda)$ with $\{p, P, \dot{f}\} \in N$. For each $n$, let

$$
D_{n}=\{r \in P \text { : for some } x \in V, r \Vdash \dot{f}(n)=x\} .
$$

Note that $D_{n}$ is dense open in $P$ as the range of $\dot{f}$ consists of ground model elements, and clearly $D_{n} \in N$.

Let $q \leq p$ be totally $(N, P)$-generic. For each $n$, choose $r_{n} \in N \cap D_{n}$ with $q \leq r_{n}$. Let $x_{n} \in V$ be such that $r_{n} \Vdash \dot{f}(n)=x_{n}$. If we define $f$ (in $V$ ) by $f(n)=x_{n}$, then $q \Vdash \dot{f}=f$ as desired.

The implication $(2) \rightarrow(3)$ is trivial, so we show next that (3) implies (1).

In regard to this, let relevant $N$ and $p$ be given, and let $\left\{D_{n}: n \in \omega\right\}$ enumerate the dense open subsets of $P$ that are in $N$. Fix a function $g: \omega \times \omega \rightarrow P$ so that

$$
D_{n} \cap N=\{g(m, n): m \in \omega\}
$$

and let $r \leq p$ be $(N, P)$-generic. This means that for all dense open $D_{n} \in N, D_{n}$ is predense below $r$; that is, for all $s \leq r$ there exists $t \in D_{n} \cap N$ such that $s$ and $t$ are compatible. Since $r \Vdash N \cap D_{n} \cap \dot{G}_{P} \neq \emptyset$, there is a $P$-name $\dot{f}$ such that

$$
r \Vdash \forall n\left[\dot{f}(n) \text { is the least } m \text { such that } g(m, n) \in \dot{G}_{P}\right] \text {. }
$$

Since forcing with $P$ adds no new reals, there is a function $f \in \omega^{\omega}$ (in $\mathrm{V}$ ) and $q \leq r$ such that $q \Vdash \dot{f}=f$. Now since $q$ is an extension of $r$, we know that for each $n$, $q$ forces that $g(f(n), n)$ is in $\dot{G}_{P}$. Since $P$ is separative, this means that $q$ actually extends $g(f(n), n)$, and so $q$ is totally $(N, P)$-generic. Note that it is important that $g(f(n), n)$ is an honest-to-goodness member of $P$, and not just a $P$-name like $g(\dot{f}(n), n)$.

We remark that the notion of forcing, shooting a closed unbounded set through a stationary, co-stationary $S \subseteq \omega_{1}$ using countable conditions is an example of a notion of forcing that adds no new functions from $\omega$ to $V$ that fails to be proper, and that Namba forcing (which changes the cofinality of $\aleph_{2}$ to $\aleph_{0}$ ) is an example of a notion of forcing that adds no new reals and yet adds a new function from $\omega$ to $V$.

Given a countable $N \prec H(\lambda)$ containing a notion of forcing $P$ and a $\bar{G}$ in $\operatorname{Gen}(N, P)$, we may ask what happens if we interpret the $P$-names that are in $N$ using $\bar{G}$ as an oracle. In general, $\bar{G}$ will not have enough information to give us a complete interpretation of $\dot{\tau}$. For example, if $\dot{G}$ is the canonical $P$-name for the 
generic object, then $\dot{G}[\bar{G}]$ is just $\bar{G}$ - a truncated picture of what $\dot{G}$ will look like in a real generic extension. On the other hand, if we look at $\check{\omega}_{1}[\bar{G}]$ (where $\check{\omega}_{1}$ is the canonical name for $\omega_{1}$ ), we see that $\check{\omega}_{1}[\bar{G}]=\omega_{1}$ - no truncation at all. The problem here is that $N$ is not transitive; things are much nicer if we look at the Mostowski collapse of $N$ and $\bar{G}$.

Definition 3.4. Let $P$ be a notion of forcing, and let $N$ be a countable elementary submodel of $H(\lambda)$ that contains $P$.

1. If $G$ is a generic subset of $P$, then in $V[G]$ let

$$
N[G]=\{\dot{\sigma}[G]: \dot{\sigma} \text { a } P \text {-name in } N\} .
$$

2. $N[\dot{G}]$ is a $P$-name forced by every condition to be the set $N[G]$ defined above.

3. If $\bar{G}$ is in $\operatorname{Gen}(N, P)$ and $\dot{\sigma}$ is a $P$-name from $N$, then we define $\dot{\sigma}[\bar{G}]$ by setting

$$
\dot{\sigma}[\bar{G}]=\pi(\dot{\sigma})[\pi(\bar{G})]
$$

where $\pi$ is the Mostowski map taking $\mathcal{P}(N)$ to its transitive collapse.

4. If $\bar{G}$ is in $\operatorname{Gen}(N, P)$, then let

$$
N[\bar{G}]=\{\dot{\sigma}[\bar{G}]: \dot{\sigma} \text { a } P \text {-name in } N\} .
$$

The first thing one should note is that $N[\bar{G}]$ is in the ground model, whereas $N[G]$ exists only in a generic extension. Assume now that our notion of forcing is totally proper, $\bar{G} \in \mathrm{Gen}^{+}(N, P)$, and $G$ is a generic subset of $P$ containing a lower bound $q$ for $\bar{G}$. Since $q$ is totally $(N, P)$-generic, we know that $N[G]$ is a countable elementary submodel of $H(\lambda)[G]$ and $N \cap G=\bar{G}$ (see Lemma 3.6 below). In $V[G]$, if we take the transitive collapse of $N[G]$, we get $N[\bar{G}]$. Thus even though we need all of $G$ to get $N[G]$, the collapse of $N[G]$ depends only on $\bar{G}$, and any lower bound $q$ for $\bar{G}$ forces that $N[\bar{G}]$ (in the ground model) is the transitive collapse of $N[\dot{G}]$ (in the extension).

Lemma 3.5. Suppose $P$ is totally proper, $N$ is a countable elementary submodel of some $H(\lambda), p \in N$, and $\bar{G} \in \operatorname{Gen}^{+}(N, P)$ with lower bound $r$. Then for a formula $\psi\left(x_{0}, \ldots, x_{n}\right)$ and $P-$ names $\dot{\sigma}_{0}, \ldots, \dot{\sigma}_{n}$ from $N$, the following are equivalent:

1. $N[\bar{G}] \models \psi\left(\dot{\sigma}_{0}[\bar{G}], \ldots, \dot{\sigma}_{n}[\bar{G}]\right)$,

2. $r \Vdash N[\dot{G}] \models \psi\left(\dot{\sigma}_{0}, \ldots, \dot{\sigma}_{n}\right)$,

3. $r \Vdash \psi\left(\dot{\sigma}_{0}, \ldots, \dot{\sigma}_{n}\right)$.

Proof. Clearly (2) if and only if (3) because $r$ forces that $N[\dot{G}]$ is an elementary submodel of $H(\lambda)[\dot{G}]$. For (1) if and only if (2), let $G$ be any generic subset of $P$ that contains $r$. In $V[G]$, the Mostowski collapse of $N[G]$ onto $N[\bar{G}]$ is an isomorphism of models, and by definition, $\pi\left(\dot{\sigma}_{i}[G]\right)=\dot{\sigma}_{i}[\bar{G}]$.

Notice in the above lemma that statement 1 does not depend on the lower bound $r$ for $\bar{G}$. Thus if we verify that $N[\bar{G}] \models \psi$, then we know every lower bound for $\bar{G}$ forces $\psi$ to be true in the extension.

Lemma 3.6. If $\bar{G} \in \operatorname{Gen}(N, P)$, then $\bar{G}$ is a maximal filter on $N \cap P$.

Proof. This is immediate, because for each $p \in N \cap P$, the set of $q$ that either extends $p$ or is incompatible with $p$ is dense in $P$ and a member of $N$, hence $\bar{G}$ contains such a $q$. 
Note that as a corollary to the above lemma, if $\bar{G} \in \mathrm{Gen}^{+}(N, P)$ and $r$ is a lower bound for $\bar{G}$, then $r \Vdash N \cap \dot{G}_{P}=\bar{G}$. Even though there are lots of dense subsets of $P$ that are not in $N, \dot{G}_{P} \cap N$ cannot grow any larger than $\bar{G}$. The above lemma is just a "local version" of the well-known fact that generic filters on a partial order must be maximal.

Lemma 3.7. If $P$ is totally proper and $G \subseteq P$ is generic, then $G$ is downward $\sigma$-directed, i.e., given $\left\{p_{n}: n \in \omega\right\} \subseteq G$, there is a $p \in G$ such that $\forall n\left[p \leq p_{n}\right]$.

Proof. In $V[G]$, let $\left\{p_{n}: n \in \omega\right\}$ be given. By Proposition 3.3, we know that $\left\{p_{n}: n \in \omega\right\} \in V$. Let $D$ be the set of $q \in p$ that is either a lower bound for $\left\{p_{n}: n \in \omega\right\}$ or incompatible with some $p_{n}$. Since $P$ is separative, this set is dense open in $P$. Since $D \in V$, there is some $q \in D \cap G$. Now the fact that $G$ is a filter means that $q$ must be a lower bound for $\left\{p_{n}: n \in \omega\right\}$.

Lemma 3.8. Let $P$ be totally proper, and let $N \in M$ be countable elementary submodels of $H(\lambda)$ with $P \in N$. If $r$ is both $(N, P)$-generic and $(M, P)$-generic, then $r \Vdash N \cap \dot{G} \in \operatorname{Gen}^{+}(N, P) \cap M$.

Proof. Let $G$ be any generic subset of $P$ that contains $r$. Since $r$ is $(N, P)$-generic, we know $N \cap G \in \operatorname{Gen}(N, P)$, and by the downward $\sigma$-directedness of $G$ we can find a lower bound for $N \cap G$. Thus we need only verify that $N \cap G$ is a member of $M$. To see this, define

$$
D=\{p \in P: p \text { is totally }(N, P) \text {-generic, }
$$

or $p$ has no totally $(N, P)$-generic extension $\}$.

The set $D$ is a member of $M$ and is dense and open in $P$. Since $r \in G$ is $(M, P)_{-}^{-}$ generic, we know that there is some $q$ in $M \cap D \cap G$. By the directedness of $G, q$ has a totally $(N, P)$-generic extension and so $q$ must be $(N, P)$-generic. By Lemma 3.6, $N \cap G=\{p \in N \cap P: q \leq p\}$, and so $N \cap G$ is definable from parameters in $M$.

One other strengthening of properness is relevant to our proof, so we need a few more definitions.

Definition 3.9. We say $\mathfrak{N}=\left\langle N_{\xi}: \xi \leq \alpha\right\rangle$ is an $\alpha$-tower if for some large enough regular cardinal $\lambda$, each $N_{\xi}$ is a countable elementary submodel of $H(\lambda)$, the sequence $\mathfrak{N}$ is increasing and continuous at limits, and $\left\langle N_{\zeta}: \zeta \leq \xi\right\rangle \in N_{\xi+1}$. A notion of forcing $P$ is said to be $\alpha$-proper if for every $\alpha$-tower $\mathfrak{N}$ such that $P \in N_{0}$, and for every $p \in N_{0} \cap P$, there is a $q \leq p$ that is $\left(N_{\xi}, P\right)$-generic for each $\xi \leq \alpha$. We say $P$ is $<\omega_{1}$-proper if $P$ is $\alpha$-proper for each $\alpha<\omega_{1}$.

Iterations of $<\omega_{1}$-proper notions of forcing are dealt with in Chapter $\mathrm{V}$ of [5]; the same basic iteration theorems that are true for proper forcing, hold in the $<\omega_{1}$-proper case as well, and we make use of this in the sequel.

\section{One space at a time}

Let $X$ be a locally compact sub-Ostaszewski space. We may assume that $\omega_{1}$ is the underlying set of $X$ and that each initial segment of $\omega_{1}$ is open in $X$. The forcing notion defined below is based on work of Abraham and Todorcevic [1] as presented in [2]. 
Definition 4.1. Our notion of forcing $P_{X}$ consists of pairs $p=\left(x_{p}, \Phi_{p}\right)$ where

1. $x_{p}: \delta \rightarrow 2$ for some $\delta<\omega_{1}$;

2. $[p]=x_{p}^{-1}(\{1\})$ has compact closure in $X$;

3. $\Phi_{p}$ is a countable set of functions $f$ such that

(a) $\operatorname{dom}(f)$ is an uncountable subset of $\omega_{1}$,

(b) for $\alpha \in \operatorname{dom}(f), f(\alpha)$ is a compact open neighborhood of $\alpha$ and $f(\alpha) \subseteq$ $\alpha+1$.

Such a function $f$ is called a promise. A condition $q$ extends a condition $p$ (written $q \leq p)$ if

4. $x_{q} \supseteq x_{p}$

5. $\Phi_{q} \supseteq \Phi_{p}$

6. for every $f \in \Phi_{p}$, the set $Y(f, p, q)=\{\xi \in \operatorname{dom}(f):[q] \backslash[p] \subseteq f(\xi)\}$ is uncountable, and $f\left\lceil Y(f, p, q) \in \Phi_{q}\right.$.

A condition $p$ should be viewed as an approximation of a subset $X^{\prime}$ of $X$ (this is $[p])$ together with some restraints on how this approximation can be extended (this is $\Phi_{p}$ ). An $f \in \Phi_{p}$ promises that when we add new points to [p], they will be contained in $f(\alpha)$ for uncountably many $\alpha \in \operatorname{dom}(f)$. If we can show that this set $X^{\prime}$ adjoined by $P_{X}$ is uncountable in the extension, then $X$ is no longer hereditarily separable because each countable subset of $X^{\prime}$ will have compact (hence countable) closure by condition 2 above. Since sub-Ostaszewski spaces are hereditarily separable, we know that $X$ will no longer be a sub-Ostaszewski space in the extension.

Lemma 4.2. For every $p \in P_{X}$ and $\theta<\omega_{1}$, there is an extension $q$ of $p$ with $[q] \backslash \theta \neq \emptyset$.

Proof. Assume this is not the case, and fix $p \in P_{X}$ and $\theta<\omega_{1}$ such that every extension $q$ of $p$ has $[q] \subseteq \theta+1$. Now if $\alpha>\theta$, the only obstacle to adding $\alpha$ to $[p]$ is that there is some promise $f_{\alpha} \in \Phi_{p}$ such that $\left\{\xi \in \operatorname{dom}\left(f_{\alpha}\right): \alpha \in f_{\alpha}(\xi)\right\}$ is countable. Since $\Phi_{p}$ is countable, there is an uncountable set $Z \subseteq \omega_{1} \backslash \theta$ and a single $f \in \Phi_{p}$ such that

$$
\forall \alpha \in Z\{\xi \in \operatorname{dom}(f): \alpha \in f(\xi)\} \text { is countable. }
$$

Let $Y_{0}=\operatorname{dom}(f) \cap \operatorname{cl} Z$. Since cl $Z$ is co-countable, we know that $Y_{0}$ is uncountable. Since $X$ is hereditarily separable, there is a countable subset $Z_{0}$ of $Z$ such that $\operatorname{cl} Z_{0}=\operatorname{cl} Z$. Given $\beta \in Y_{0}$, there is some $z_{\beta} \in Z_{0}$ with $z_{\beta} \in f(\beta)$. Since $Y_{0}$ is uncountable and $Z_{0}$ is countable, there is a $z \in Z_{0}$ such that $\left\{\beta \in Y_{0}: z \in f(\beta)\right\}$ is uncountable. This is a contradiction of (4.1) as $z \in Z$ and $Y_{0} \subseteq \operatorname{dom}(f)$.

Lemma 4.2 tells us that the set adjoined by $P_{X}$ is cofinal in $\omega_{1}^{V}$, but we have yet to show that $\omega_{1}^{V}$ is still uncountable in the extension. The remainder of this section will establish that $P_{X}$ is totally proper, and hence $\omega_{1}$ is preserved. To start, we need a definition.

Definition 4.3. Given a condition $p \in P_{X}$ and a dense open subset $D$ of $P_{X}$, define

$$
\begin{aligned}
& \operatorname{Bad}(p, D)=\left\{\alpha \in \omega_{1} \backslash \operatorname{dom}\left(x_{p}\right) \text { : there is a compact open } U_{\alpha}\right. \\
& \text { with } \left.\alpha \in U_{\alpha} \subseteq \alpha+1 \text { such that whenever } q \leq p \text { and } q \in D,[q] \backslash[p] \nsubseteq U_{\alpha}\right\} .
\end{aligned}
$$

Lemma 4.4. For $p \in P_{X}$ and $D \subseteq P$ dense open, $\operatorname{Bad}(p, D)$ is countable. 
Proof. Suppose this is not the case. Define a function $f$ with domain $\operatorname{Bad}(p, D)$ and for $\alpha \in \operatorname{dom}(f), f(\alpha)$ is a compact open set witnessing that $\alpha \in \operatorname{Bad}(p, D)$. Since $\operatorname{Bad}(p, D)$ is uncountable, $f$ is a promise and thus $r=\left(x_{p}, \Phi_{p} \cup\{f\}\right)$ is a condition in $P_{X}$. Because $[r] \backslash[p]=\emptyset, r$ is an extension of $p$. Now $D$ is dense in $P_{X}$ so we can find a $q \in D$ that extends $r$. The definition of extension in $P_{X}$ implies that $Y=\{\alpha \in \operatorname{Bad}(p, D):[q] \backslash[r] \subseteq f(\alpha)\}$ is uncountable. Since $[q] \backslash[r]=[q] \backslash[p]$, we know that if $\alpha \in Y$, then $[q] \backslash[p] \subseteq f(\alpha)$, contradicting the fact that $f(\alpha)$ witnesses that $\alpha \in \operatorname{Bad}(p, D)$.

Lemma 4.5. Fix a countable $N \prec H(\lambda)$ with $X$ and $P_{X}$ in $N$, and let $U$ be an open subset of $X$ with $U \cap N$ unbounded in $\delta=N \cap \omega_{1}$. Given a condition $p \in N \cap P_{X}$ and a dense open subset $D$ of $P_{X}$ that is in $N$, there is an extension $q$ of $p$ such that $q \in N \cap D$ and $[q] \backslash[p] \subseteq U$.

Proof. All parameters involved in the definition of $\operatorname{Bad}(p, D)$ are members of $N$, so $\operatorname{Bad}(p, D) \in N$ as well. Since $\operatorname{Bad}(p, D)$ is countable, we know it must be bounded below $\delta$, and since $U \cap \delta$ is cofinal in $\delta$, we can find a $\beta<\delta$ such that $\beta \in$ $U \backslash \operatorname{Bad}(p, D)$. By our comments above, we can find a compact open neighborhood $V$ of $\beta$ such that $V \subseteq \beta+1 \cap U$ and $V \in N$. Since $\beta \notin \operatorname{Bad}(p, D)$, in $N$ we can find an extension $q$ of $p$ such that $q \in D$ and $[q] \backslash[p] \subseteq V \subseteq U$ as promised.

We are almost in a position to show that $P_{X}$ is totally proper-given a relevant countable model $N$ and a condition $p \in N \cap P_{X}$, we can fix some compact open neighborhood $U$ of $\delta=N \cap \omega_{1}$ and repeatedly apply the above lemma to build a decreasing sequence $p=p_{0} \geq p_{1} \geq \ldots$ of conditions in $N \cap P_{X}$ such that $\left[p_{n}\right] \backslash[p] \subseteq U$ for each $n$ and if $D \in N$ is dense open in $P_{X}$, then some $p_{n}$ is in $D$. The set $\bigcup_{n \in \omega}\left[p_{n}\right]$ is contained in $U$ modulo a compact set, and hence it will have compact closure. This is not quite enough to ensure that the sequence of $p_{n}$ 's has a lower bound - somehow we need to make sure that if $f$ is a promise appearing in some $\Phi_{p_{n}}$ then

$$
\bigcap_{m>n} Y\left(f, p_{m}, p_{n}\right) \text { is uncountable }
$$

because the lower bound has to keep all promises that have arisen along the way. We need another definition.

Definition 4.6. If $f$ is a promise, define

$$
\begin{aligned}
& H_{f}=\left\{\alpha<\omega_{1}: \text { there is a set } V_{\alpha}\right. \text { that is open, cofinally convergent, } \\
& \text { and cofinal in } \alpha \text { with compact closure such that the set } \\
& \left.\qquad K_{\alpha, f}=\left\{\beta \in \operatorname{dom}(f): \operatorname{Top}\left(V_{\alpha}\right) \in f(\beta)\right\} \text { is countable }\right\} .
\end{aligned}
$$

Lemma 4.7. For any promise $f$, the set $H_{f}$ is countable.

Proof. Suppose this is not the case. Since $\alpha \leq \operatorname{Top}\left(V_{\alpha}\right)$ for each $\alpha \in H_{f}$, the set $Y=\left\{\operatorname{Top}\left(V_{\alpha}\right): \alpha \in H_{f}\right\}$ is uncountable, and hence $\operatorname{cl} Y$ is co-countable. Choose $\xi$ large enough so that $\omega_{1} \backslash \xi \subseteq \operatorname{cl} Y=\operatorname{cl}(Y \cap \xi)$, and choose a $\gamma \in \operatorname{dom}(f)$ such that $\gamma>\xi$ and $\sup K_{\zeta, f}<\gamma$ for all $\zeta \in H_{f} \cap \gamma$. But then $Y \cap \xi \cap f(\gamma)=\emptyset$, contradicting the density of $Y \cap \xi$ in $\operatorname{cl} Y$.

Lemma 4.8. Let $N \prec H(\lambda)$ be countable with $X$ and $P_{X}$ in $N$. Let $U$ be a cofinally convergent open set, cofinal in $\delta=N \cap \omega_{1}$, and let $f \in N$ be a promise. Then there 
is a compact open $V_{f}$ bounded below $\delta$ such that $\left\{\alpha \in \operatorname{dom}(f): U \backslash f(\alpha)=V_{f}\right\}$ is uncountable.

Proof. Since $H_{f}$ is countable and $H_{f} \in N$, we know that $\delta \notin H_{f}$ and thus

$$
K_{\delta, f}=\{\beta \in \operatorname{dom}(f): \operatorname{Top}(U) \in f(\beta)\}
$$

is uncountable. Now $U$ is cofinally convergent so by Lemma 4.8, $\operatorname{Top}(U) \in f(\beta)$ if and only if there is a compact open $W$ bounded below $\delta$ such that $U \backslash f(\beta)=W$. Since $U$ has only countably many compact open subsets, there is a single $V_{f}$ such that $\left\{\alpha \in \operatorname{dom}(f): U \backslash f(\alpha)=V_{f}\right\}$ is uncountable.

Lemma 4.9. Given $N \prec H(\lambda)$ countable with $X$ and $P_{X}$ in $N, p \in N \cap P_{X}$, and $U$ cofinally convergent, compact open and cofinal in $\delta=N \cap \omega_{1}$, there is an extension $q$ of $p$ such that $q$ is totally $\left(N, P_{X}\right)$-generic and $[q] \backslash[p] \subseteq U$.

Proof. Fix an enumeration $\left\{D_{n}: n \in \omega\right\}$ of the dense open subsets of $P_{X}$ that are members of $N$. By iterating Lemma 4.5, we can certainly construct a decreasing sequence of conditions $\left\{p_{n}: n \in \omega\right\}$ starting with $p_{0}=p$ such that $p_{n+1} \in N \cap D_{n}$ and $\left[p_{n+1}\right] \backslash\left[p_{n}\right] \subseteq U$. Let $x=\bigcup_{n \in \omega} x_{p_{n}}$ and $[x]=x^{-1}(\{1\})$. Since $[x] \backslash[p] \subseteq U$, we know that $[x]$ has compact closure. If the sequence $\left\{p_{n}: n \in \omega\right\}$ is said to have a lower bound, then for each $n \in \omega$ and each promise $f \in \Phi_{p_{n}}$, we need for there to be uncountably many $\xi$ in $\operatorname{dom}(f)$ with $[x] \backslash\left[p_{n}\right] \subseteq f(\xi)$.

To ensure this, we make sure that every $f$ that appears in some $\Phi_{p_{n}}$ gets handled in the following manner:

Assume we have defined $p_{n}$ and we are given an $f \in \Phi_{p_{i}}$ for some $i \leq n$ that must be handled at this stage. First, we know that the set

$$
Y=\left\{\xi \in \operatorname{dom}(f):\left[p_{n}\right] \backslash\left[p_{i}\right] \subseteq f(\xi)\right\}
$$

is uncountable and $f \uparrow Y \in \Phi_{p_{n}}$. Since $f \uparrow Y$ is in $N$, we know by Lemma 4.8 that there is a compact open $V$ bounded below $\delta$ such that the set $Z=\{\xi \in Y$ : $U \backslash f(\xi)=V\}$ is uncountable. Let $s$ be an extension of $p_{n}$ with $V \subseteq \operatorname{dom}\left(x_{s}\right)$ defined by setting $x_{s}(\xi)=0$ on any $\xi$ added to $\operatorname{dom}\left(x_{p_{n}}\right)$. Since $V$ is bounded below $\delta$, we can find such an $s$ in $N$. We can apply Lemma 4.5 to get a $p_{n+1} \leq s$ such that $p_{n+1} \in N \cap D_{n}$ and $\left[p_{n+1}\right] \backslash[s]=\left[p_{n+1}\right] \backslash\left[p_{n}\right] \subseteq U$. Notice that if $r$ is any extension of $p_{n+1}$ that satisfies $[r] \backslash\left[p_{n+1}\right] \subseteq U$, then $[r] \backslash\left[p_{n+1}\right] \subseteq f(\xi)$ for all $\xi \in Z$.

Since each condition involves only countably many promises, we can construct $\left\{p_{n}: n \in \omega\right\}$ so that

1. $p_{0}=p, p_{n+1} \leq p_{n}, p_{n+1} \in N \cap D_{n}$;

2. for each $n$ and $f \in \Phi_{p_{n}}$, there is some later stage $n_{f}$ such that $f$ is handled at stage $n_{f}$ in the manner described above.

Once this is accomplished, let $x=\bigcup_{n \in \omega}$ and $[x]=x^{-1}(\{1\})$. We claim that if $f \in \Phi_{p_{n}}$ for some $n$, then $Y_{f}=\left\{\xi \in \operatorname{dom}(f):[x] \backslash\left[p_{n}\right] \subseteq f(\xi)\right\}$ is uncountable. To see this, fix $m \geq n$ such that $f$ was handled when we defined $p_{m+1}$. Let

$$
Y=\left\{\xi \in \operatorname{dom}(f):\left[p_{m}\right] \backslash\left[p_{n}\right] \subseteq f(\xi)\right\} .
$$

We fixed $V$ such that $Z=\{\xi \in Y: U \backslash f(\xi)=V\}$ is uncountable, and by our comments above, we know that for all $\xi \in Z$ and $i>m$ that $\left[p_{i}\right] \backslash\left[p_{m+1}\right] \subseteq f(\xi)$, 
so $Z \subseteq Y_{f}$. Thus if we define $q$ by

$$
q=\left(x, \bigcup_{n \in \omega} \Phi_{p_{n}} \cup\left\{f \uparrow Y_{f}: f \in \bigcup_{n \in \omega} \Phi_{p_{n}}\right\}\right),
$$

then $q$ is a lower bound for the sequence $\left\{p_{n}: n \in \omega\right\}$ in $P_{X}$.

Theorem 2 of the Introduction now follows as a trivial corollary of Lemma 4.9. A similar proof shows that $P_{X}$ is in fact $<\omega_{1}$-proper. Since this will be needed in the sequel, we present this now.

Proposition 4.10. If $X$ is an Ostaszewski space, the forcing $P_{X}$ defined above is $<\omega_{1}$-proper.

Proof. We prove by induction on $\alpha<\omega_{1}$ that for each countable $\alpha$-tower $\mathfrak{N}=$ $\left\langle N_{\xi}: \xi \leq \alpha\right\rangle$ with $P_{X} \in N_{0}$, any $p \in N_{0} \cap P_{X}$, and any open $U$ open with compact closure and cofinal in $\delta_{\alpha}=N_{\alpha} \cap \omega_{1}$ that there is a $q \leq p$ with $[q] \backslash[p] \subseteq U$ that is totally $\left(N_{\xi}, P_{X}\right)$-generic for each $\xi \leq \alpha$. The case where $\alpha$ is a successor ordinal follows from the induction hypothesis by a simple application of Lemma 4.9, so we assume that $\alpha$ is a limit ordinal.

Fix an increasing sequence $\left\{\alpha_{n}: n \in \omega\right\}$ cofinal in $\alpha$. We construct a decreasing sequence $\left\{p_{n}: n \in \omega\right\}$ satisfying that

1. $p_{0}=p, p_{n+1} \in N_{\alpha_{n}+1}$ is totally $\left(N_{\xi}, P_{X}\right)$-generic for $\xi \leq \alpha_{n}$,

2. $\left[p_{n+1}\right] \backslash\left[p_{n}\right] \subseteq U$,

3. each promise $f$ appearing in some $\Phi_{p_{n}}$ gets handled at some stage in the manner described below.

Assume we have $p_{n}$ satisfying 1 and 2 above, and we are given a promise $f$ from some $\Phi_{p_{i}}$ where $i \leq n$. We know that $Y\left(f, p_{i}, p_{n}\right)$ is uncountable, and $g=f \uparrow$

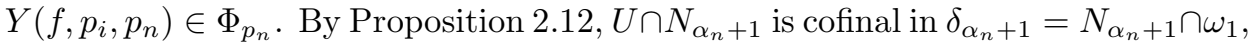
so there is a compact open $V_{g}$ bounded below $\delta_{\alpha_{n+1}}$ such that

$$
Z_{g}=\left\{\xi \in \operatorname{dom}(g): U \backslash g(\xi)=V_{g}\right\}
$$

is uncountable.

In $N_{\alpha_{n}+1}$, extend $p_{n}$ to a condition $s$ satisfying $\left[p_{n}\right]=[s]$ and $V_{g} \subseteq \operatorname{dom}\left(x_{s}\right)$, and then apply our induction hypotheses to $s, U$, and the tower $\left\langle N_{\xi}: \alpha_{n}<\xi \leq \alpha_{n+1}\right\rangle$ to get $p_{n+1} \in N_{\alpha_{n+1}+1}$ as needed. Just as in the proof of Lemma 4.9 , if $r \leq p_{n+1}$ satisfies $[r] \backslash\left[p_{n+1}\right] \subseteq U$, then $Z_{g} \subseteq Y\left(g, r, p_{n+1}\right)$, and an argument almost identical to the one given there shows us that $\left\{p_{n}: n \in \omega\right\}$ has a lower bound $q$ which is $\left(N_{\xi}, P_{X}\right)$-generic for $\xi \leq \alpha$ and $[q] \backslash[p] \subseteq U$.

\section{TWO STEP ITERATIONS}

Now that we know we can destroy an Ostaszewski space without adding new reals, the next problem is to show that an iteration of such forcings does not add reals.

To start our discussion of this problem, assume that $P$ is a totally proper notion of forcing and $\dot{Q}$ is a $P$-name for a totally proper notion of forcing. Fix a countable elementary submodel $N$ of some $H(\lambda)$ that contains both $P$ and $\dot{Q}$. The question we need to address is: If $r$ is totally $(N, P)$-generic, can we find a $P$-name $\dot{s}$ so that $r * \dot{s}$ is totally $(N, P * \dot{Q})$-generic?

The obvious thing to try is to choose $\dot{s}$ such that

$$
r \Vdash \dot{s} \text { is totally }\left(N\left[\dot{G}_{P}\right], \dot{Q}\right) \text {-generic. }
$$


If $D \in N$ is dense and open in $P * \dot{Q}$, then certainly

$$
r * \dot{s} \Vdash \text { there is a } t \in N \cap D \text { such that } r * \dot{s} \leq t
$$

but $r * \dot{s}$ need not be an extension of any particular $t \in N \cap D$, and hence not totally $(N, P * \dot{Q})$-generic.

What we need is a sequence $\left\{\dot{q}_{n}: n \in \omega\right\}$ of $P$-names from $N$ (which is a stronger requirement than $\left.r \Vdash \dot{q}_{n} \in N[\dot{G}]\right)$ so that

$$
r \Vdash\left\{\dot{q}_{n}: n \in \omega\right\} \text { generates a member of } \mathrm{Gen}^{+}(N[\dot{G}], \dot{Q}) \text {. }
$$

If $\dot{s}$ is forced by $r$ to extend each $\dot{q}_{n}$, then it is not hard to see that $r * \dot{s}$ is totally $(N, P * \dot{Q})$-generic. Thus we need to address the question: What does it take for $r$ to see such a sequence of $P$-names?

In the situation of interest to us, namely when there is a $P$-name $\dot{X}$ for an Ostaszewski space and $\dot{Q}$ is a name for the forcing $P_{\dot{X}}$ introduced in the previous section, we can get a reasonable condition on $r$ that guarantees the existence of a suitable $\dot{s}$.

We will actually need something a bit stronger than this in the proof of our iteration theorem, but in order to see where we are headed, we need another definition.

Definition 5.1. Assume that

1. $P$ is totally proper, and $\Vdash_{P} \dot{Q}$ is totally proper,

2. $N \prec H(\lambda)$ is countable with $\{P, \dot{Q}\} \in N$,

3. $\bar{G} \in \operatorname{Gen}(N, P)$,

Then $\bar{H} \in \operatorname{Gen}(N[\bar{G}], \dot{Q})$ means

4. $\bar{H}$ consists of $P$-names from $N$,

5. $\{\dot{s}[\bar{G}]: \dot{s} \in \bar{H}\}$ is an $N[\bar{G}]$-generic subset of $\dot{Q}[\bar{G}]$.

Similarly, if $\dot{q} \in N$ is a $P$-name for an element of $\dot{Q}$, then we let

$$
\operatorname{Gen}(N[\bar{G}], \dot{Q}, \dot{q})=\{\bar{H} \in \operatorname{Gen}(N[\bar{G}], \dot{Q}): \dot{q} \in \bar{H}\} .
$$

Now our goal is to prove the following theorem which will be crucial in our proof that our forcing can be iterated without adding reals.

Theorem 3. Assume

1. $P$ is totally proper,

2. $\dot{X}$ is a $P$-name for an Ostaszewski space,

3. $\dot{Q}$ is a $P$-name for $P_{\dot{X}}$, the notion of forcing that destroys $\dot{X}$,

4. $N_{0} \in N_{1} \in N_{2}$ are countable elementary submodels of $H(\lambda)$,

5. $\{P, \dot{X}, \dot{Q}\} \in N_{0}$,

6. $\bar{G} \in \mathrm{Gen}^{+}\left(N_{0}, P\right) \cap N_{1}$,

7. $\dot{q} \in N$ is a $P$-name for a condition in $\dot{Q}$.

Then we can find $\bar{H} \in \operatorname{Gen}\left(N_{0}[\bar{G}], \dot{Q}, \dot{q}\right)$ so that whenever $r$ is a lower bound for $\bar{G}$ that is $\left(N_{i}, P\right)$-generic for $i=1,2$, we have

$$
r \Vdash \bar{H} \text { has a lower bound in } \dot{Q} \text {. }
$$

Notice the order of the quantifiers here - we build a single $\bar{H}$ that works for all relevant $r$. The proof of this will appear later in the section. Even though it may seem hopeless to build such an $\bar{H}$ that takes into account all of the relevant $r$, the key is Lemma 3.8. All $r$ of concern to us have the same general idea of what $N_{1} \cap \dot{G}$ might be — they all agree it must lie in $N_{2} \cap \operatorname{Gen}\left(N_{1}, P\right)$. 
We add to the proof of Theorem 3 by considering our situation in detail. Assume that $P$ is a totally proper notion of forcing and $N$ is a countable elementary submodel of some $H(\lambda)$ such that $P \in N$. Further assume that $\dot{X} \in N$ is a name forced by every condition in $P$ to be an Ostaszewski space with underlying set $\omega_{1}$ and initial segments open.

If $\bar{G}$ is an element of $\mathrm{Gen}^{+}\left(N_{0}, P\right)$ and $\alpha \in N$ is a countable ordinal, then there is a $P$-name $\dot{\tau}_{\alpha}$ for a countable family of subsets of $\alpha+1$ such that every condition in $P$ forces $\dot{\tau}_{\alpha}$ is a base for the topology of $\dot{X}$ at $\alpha$. Because $P$ is totally proper, $\dot{\tau}_{\alpha}$ is forced to be an element of the ground model, and thus some condition in $\bar{G}$ decides a specific value for $\dot{\tau}_{\alpha}$.

If we let $\delta=N \cap \omega_{1}$, then by decoding all the names $\dot{\tau}_{\alpha}$ using $\bar{G}$ we get a first countable, locally countable topology on $\delta$, and any lower bound for $\bar{G}$ forces that this is in fact the topology on $\delta$ viewed as a subspace of $\dot{X}$. We let $X_{\delta}$ denote this topology that we get from $\bar{G}$.

Let us step, for a moment, into the model $N[\bar{G}]$ defined as in Definition 3.4. As far as $N[\bar{G}]$ is concerned $\dot{X}[\bar{G}]$ is an Ostaszewski space, and in fact $\dot{X}[\bar{G}]=X_{\delta}$. This follows because in the generic extension, the Mostowski collapse of $N[G]$ (where $G$ is any generic subset of $P$ containing a lower bound for $\bar{G}$ ) is the identity map when restricted to the hereditarily countable sets.

We now have the background necessary to prove Theorem 3 , but it is instructive at this point to consider first a simplified version of our goal.

Definition 5.2. In the situation of Theorem 3, we say $r \in P$ is generic enough if $r$ is a lower bound for $\bar{G}$ that is $\left(N_{i}, P\right)$-generic for $i=1,2$.

Proposition 5.3. In the situation of Theorem 3, we can find a set $V$ open in $X_{\delta}$ such that any generic enough $r$ will force $V$ to be cofinally convergent in $\delta$.

Proof. First, we note that the space $X_{\delta}$ is in $N_{1}$ because it is definable from $\bar{G}$ and $P$-names from $N_{0}$. Let $\left\{\bar{H}_{n}: n \in \omega\right\}$ list all members of $\operatorname{Gen}^{+}\left(N_{1}, P\right) \cap N_{2}$ that extend $\bar{G}$; since $N_{1}$ contains both $\bar{G}$ and a lower bound for $\bar{G}$, this set is non-empty. By Lemma 3.5, for each $n$

$$
N_{1}\left[\bar{H}_{n}\right] \models \dot{X}\left[\bar{H}_{n}\right] \text { is an Ostaszewski space and } X_{\delta} \text { is a subspace of } \dot{X}\left[\bar{H}_{n}\right] \text {. }
$$

Let $A_{n}$ be such that

$$
N_{1}\left[\bar{H}_{n}\right] \models A_{n}=\{U \subseteq \delta: U \text { is open and cofinally convergent in } \delta\} .
$$

Notice that each $U \in A_{n}$ is open in $X_{\delta}$ and since $P$ is totally proper, $A_{n} \cap N_{1}\left[\bar{H}_{n}\right] \subseteq$ $N_{1}$. Most importantly, Corollary 2.15 implies that whenever $U \in N_{1}$ is cofinal in $\delta$ and open in $X_{\delta}$, there is a $V \subseteq U$ such that $V \in N_{1} \cap A_{n}$. This is the only place where the countable compactness of Ostaszewski spaces is used in our proof.

Each $\dot{X}\left[\bar{H}_{n}\right]$ should be thought of as a guess at the topology on $\dot{X} \cap \delta_{1}$ (where $\left.\delta_{1}=N_{1} \cap \omega_{1}\right)$, and by Lemma 3.8, we know that any generic enough $r$ will force that one of these guesses is the correct topology in the generic extension. We construct an open $W \subseteq X_{\delta}$, cofinal in $\delta$, such that $W$ will be cofinally convergent no matter which of these guesses turns out to be the right one.

To do this, fix an increasing $\omega$-sequence $\left\{\alpha_{n}: n \in \omega\right\}$ cofinal in $\delta$. We define by recursion sets $U_{n}$ and $W_{n}$ such that

1. $U_{0}=\delta$,

2. $U_{n+1} \in N_{1} \cap A_{n}$, 
3. $U_{n+1} \subseteq U_{n} \backslash\left(W_{0} \cup \cdots \cup W_{n}\right)$,

4. $W_{n} \in N_{0}[\bar{G}], W_{n}$ compact open in $X_{\delta}, W_{n} \subseteq U_{n}, \max \left(W_{n}\right) \geq \alpha_{n}$.

We can always find $U_{n+1}$ because $U_{n} \backslash\left(W_{0} \cup \cdots \cup W_{n}\right) \in N_{1}$, so we can apply Corollary 2.15 in $N_{1}\left[\bar{H}_{n}\right]$.

Let $W=\bigcup_{n \in \omega} W_{n}$. Condition 4 ensures that $W$ is an open subset of $X_{\delta}$ that is cofinal in $\delta$.

We are still stuck with the task of showing that $W$ is forced to be cofinally convergent by any generic enough $r$. Fix such an $r$, and let $G$ be any generic subset of $P$ that contains $r$. By Lemma 3.8, we know there is some $n$ such that $N_{1} \cap G=\bar{H}_{n}$, and so $U_{n+1}$ is cofinally convergent in $\delta$ in $V[G]$. Since $W \backslash\left(W_{0} \cup\right.$ $\left.\cdots \cup W_{n}\right) \subseteq U_{n+1}, W$ is cofinally convergent in $\delta$ as well. Since $G$ was an arbitrary generic set containing $r$, we have then that $r \Vdash W$ is cofinally convergent in $\delta$.

Now in order to prove Theorem 3, we use the same strategy - if we know the correct topology on $X_{\delta}$ and we have countably many guesses at the correct topology on $\dot{X} \cap \delta_{1}$, one of which is guaranteed to be correct, we can build the desired sequence by diagonalization. Notice also that a single diagonalization argument will take care of all generic enough $r$ 's just as in the proof above. We've already seen how to guarantee the union of the first components of the $\dot{q}_{n}$ 's has compact closure, so the only added complication is all the promises that arise along the way.

Proof of Theorem 3. Let $\left\{\dot{D}_{n}: n \in \omega\right\}$ list all $P$-names from $N_{0}$ of dense open subsets of $\dot{Q}$, and let $\left\{\bar{H}_{n}: n \in \omega\right\}$ enumerate those members of $\operatorname{Gen}^{+}\left(N_{1}, P\right) \cap N_{2}$ that extend $\bar{G}$, and let $A_{n}$ be defined as in (5.5). The construction of $\left\{\dot{q}_{n}: n \in \omega\right\}$ is essentially a combination of Lemma 4.9 and Proposition 5.3.

Before commencing with the construction, we make a convention that when dealing with $P$-names $\dot{\sigma}$ from $N_{0}$, we identify $\dot{\sigma}$ and $\dot{\sigma}[\bar{G}]$. Thus when we say $\dot{q}_{n+1} \in \dot{D}_{n}$, we mean $N_{0}[\bar{G}] \models \dot{q}_{n+1}[\bar{G}] \in \dot{D}_{n}[\bar{G}]$, when we write $\left[\dot{q}_{n}\right]$, we mean $\left[\dot{q}_{n}[\bar{G}]\right]$.

We define for each $n \in \omega$ a set $U_{n}$ and a $P$-name $\dot{q}_{n} \in N_{0}$ such that

1. $U_{0}=\delta, \dot{q}_{0} \in N_{0}$ any $P$-name for a condition in $\dot{Q}$,

2. $U_{n+1} \in N_{1} \cap A_{n}$,

3. $U_{n+1} \subseteq U_{n} \backslash \operatorname{dom}\left(x_{\dot{q}_{n}}\right)$,

4. $\dot{q}_{n+1} \in \dot{D}_{n}$,

5. $\left[\dot{q}_{n+1}\right] \backslash\left[\dot{q}_{n}\right] \subseteq U_{n+1}$,

6. each promise $\dot{f}$ appearing in some $\dot{q}_{n}$ gets taken care of with respect to each $\bar{H}_{m}$ in a sense to be made precise in a moment.

At a stage $n+1$, we can choose $U_{n+1}$ satisfying 2 and 3 precisely as in the proof of the preceding proposition. Since $U_{n+1}$ is open and cofinal in $\delta$, we can apply Lemma 4.5 in $N_{1}\left[\bar{H}_{n}\right]$ to get $\dot{q}_{n+1}$ satisfying 4 and 5 .

For condition 6 , if $\dot{f}$ is a promise appearing in some $\dot{q}_{n}$ where $n>m$, then because $\bar{H}_{m}$ contains a lower bound for $\bar{G}$,

$$
N_{1}\left[\bar{H}_{m}\right] \models \dot{f}\left[\bar{H}_{m}\right] \text { is a promise in } \dot{q}_{n}\left[\bar{H}_{m}\right]
$$


In the model $N_{1}\left[\bar{H}_{m}\right]$, after the set $U_{m+1}$ has been defined, we may apply Lemma 4.8 to get a compact open $V(\dot{f}, m)$ bounded below $\delta$ such that

$$
\begin{aligned}
& N_{1}\left[\bar{H}_{m}\right] \models Z(\dot{f}, m)=\left\{\alpha \in \operatorname { d o m } \left(\dot{f}\left[\bar{H}_{m}\right]:\right.\right. \\
&\left.U_{m+1} \backslash\left(\dot{f}\left[\bar{H}_{m}\right](\alpha)\right)=V(\dot{f}, m)\right\} \text { is uncountable. }
\end{aligned}
$$

Note that Lemma 4.8 applies because $U_{m+1}$ is cofinally convergent in $N_{1}\left[\bar{H}_{m}\right]$ and $\dot{f}\left[\bar{H}_{m}\right] \in N_{0}\left[\bar{H}_{m}\right] \in N_{1}\left[\bar{H}_{m}\right]$.

When we say " $\dot{f}$ (a promise from $\dot{q}_{n}$ ) gets handled with respect to $\bar{H}_{m}$ at stage $i>m$ ", we mean when it comes time to define $\dot{q}_{i+1}$ we do the following:

In $N_{0}[\bar{G}]$, let

$$
\dot{Y}=Y\left(\dot{f}, \dot{q}_{n}, \dot{q}_{i}\right)=\left\{\xi \in \operatorname{dom}(\dot{f}):\left[\dot{q}_{i}\right] \backslash\left[\dot{q}_{n}\right] \subseteq \dot{f}(\xi)\right\} .
$$

By definition of the extension in $\dot{Q}$, we know that $\dot{Y}$ is uncountable and $\dot{f} \uparrow \dot{Y}$ is a promise in the condition $\dot{q}_{i}$. Let $\dot{s}$ be an extension of $\dot{q}_{i}$ with $[\dot{s}]=\left[\dot{q}_{i}\right]$ and $V\left(\dot{f}\lceil\dot{Y}, m)\right.$ contained in the domain of $\dot{x}_{\dot{q}_{i}}$, and then let $\dot{q}_{i+1}$ be an extension of $\dot{s}$ satisfying 4 and 5 . Note that $V(\dot{f}, m)$ was defined using the "privileged information" obtained from $\bar{H}_{m}$, but it is still a $P$-name from $N_{0}$.

Since only countably many such $\dot{f}$ appear in our construction of $\left\{\dot{q}_{n}: n \in \omega\right\}$, we can arrange that each $\dot{f}$ gets handled with respect to each $\bar{H}_{m}$ at some stage $i>m$.

Now let $r$ be generic enough, and let $G$ be any generic subset of $P$ that contains $r$. By Lemma 3.8, there is an $n$ such that $N_{1} \cap G=\bar{H}_{n}$. Thus $U_{n+1}$ is cofinally convergent and $\bigcup_{n \in \omega}\left[\dot{q}_{n}\right]$ will have compact closure. If $f$ is a promise appearing in some $\dot{q}_{n}[G]$, then $f$ is of the form $\dot{f}[G]$ for some $\dot{f} \in N_{0}$ (because $\dot{q}_{n} \in N_{0}$ contains only countably many promises), and so there is some stage $i>n$ at which $\dot{f}$ was handled with respect to $\bar{H}_{n}$. Just as in the proof of Lemma 4.9, whenever $\dot{s}$ is any extension of $\dot{q}_{n+1}$ in $N_{0}$ that satisfies $[\dot{s}] \backslash\left[\dot{q}_{n+1}\right] \subseteq U_{n+1}$, we have

$$
Y\left(\dot{f}, \dot{s}, \dot{q}_{n+1}\right) \supseteq Z(\dot{f}, n) .
$$

Since our sequence $\left\{U_{i}: i \in \omega\right\}$ is decreasing, we know that for $m \geq n+1$,

$$
\left[\dot{q}_{m}\right] \backslash\left[\dot{q}_{n+1}\right] \subseteq U_{n+1} .
$$

Thus if we let $x=\bigcup_{i \in \omega} x_{\dot{q}_{i}}$ and $[x]=x^{-1}(\{1\})$, then

$$
Y_{f}=\left\{\xi \in \operatorname{dom}(\dot{f}[G]):[x] \backslash\left[\dot{q}_{n}[G]\right] \subseteq \dot{f}[G](\xi)\right\}
$$

contains $Z(\dot{f}, n)$, and hence is uncountable.

Thus if we define $\Phi$ in $V[G]$ as the collection of all promises appearing in some $\dot{q}_{i}[G]$ together with $f \uparrow Y_{f}$ for each promise $f$ that appears in some $\dot{q}_{i}[G]$, then $q=(x, \Phi)$ is a lower bound for $\left\{\dot{q}_{i}: i \in \omega\right\}$. Since $G$ was an arbitrary generic subset of $P$ that contains $r$, we have

$$
r \Vdash\left\{\dot{q}_{n}: n \in \omega\right\} \text { generates a member of } \mathrm{Gen}^{+}\left(N_{0}[\dot{G}], \dot{Q}\right) \text {. }
$$

Thus from the sequence $\left\{\dot{q}_{n}: n \in \omega\right\}$ we get the desired $\bar{H}$. 


\section{2-COMPLETENESS AND AN ITERATION THEOREM}

The property of $P_{X}$ isolated in Theorem 3 is what allows us to prove that a countable support iteration of such forcings does not add reals. This is the motivation behind the following definition.

Definition 6.1. Let $P$ be totally proper and let $\dot{Q}$ be a $P$-name for a totally proper notion of forcing. We say $\dot{Q}$ is 2-complete for $P$ if whenever

1. $N_{0} \in N_{1} \in N_{2}$ are countable elementary submodels of $H(\lambda)$ with $P, \dot{Q} \in N_{0}$,

2. $\bar{G} \in \mathrm{Gen}^{+}\left(N_{0}, P\right) \cap N_{1}$,

3. $\dot{q} \in N_{0}$ is a $P$-name for a condition in $\dot{Q}$,

there is an $\bar{H} \in \operatorname{Gen}\left(N_{0}[\bar{G}], \dot{Q}, \dot{q}\right)$ so that whenever $r$ is a lower bound for $\bar{G}$ that is $\left(N_{i}, P\right)$-generic for $i=1,2$, we have

$$
r \Vdash \bar{H} \text { has a lower bound in } \dot{Q} \text {. }
$$

Thus Theorem 3 tells us that if $P$ is totally proper and $\dot{X}$ is a $P$-name for an Ostaszewski space, then $P_{\dot{X}}$ is 2-complete for $P$.

The proof of the following theorem is, in some sense, isomorphic to the proof of Theorem 7.1 of Chapter V in [5]. In place of Shelah's $\mathbb{D}$-completeness, a condition on individual notions of forcing, we use 2-completeness, a condition on iterations. The actual differences between our proof and Shelah's are only minor technical details — the underlying idea is unchanged.

Theorem 4. Let $\mathbb{P}=\left\langle P_{\alpha}, \dot{Q}_{\alpha}: \alpha<\kappa\right\rangle$ be a countable support iteration such that

- $\Vdash_{\alpha} \dot{Q}_{\alpha}$ is $<\omega_{1}$-proper,

- $\dot{Q}_{\alpha}$ is 2-complete for $P_{\alpha}$.

Then $P_{\kappa}$ is totally proper.

Proof. The theorem is easy if $\kappa$ is finite, so we assume that $\kappa$ is a limit ordinal. This reduction is purely for typographical convenience, since otherwise we would have to replace the $\kappa^{*}$ defined below by $2 \kappa^{*}$.

Let $N \prec H(\lambda)$ be countable with $\mathbb{P} \in N$, and let $p \in P_{\kappa} \cap N$ be arbitrary. We must produce a totally $\left(N, P_{\kappa}\right)$-generic condition $q \leq p$. For $\theta \in N \cap(\kappa+1)$ let $\theta^{*}=\operatorname{otp}(N \cap \theta)$. Also fix an elementary tower $\mathfrak{N}=\left\langle N_{\xi}: \xi \leq \kappa^{*}\right\rangle$ such that $N=N_{0}$. In order to facilitate the statment of the following claim, we let $N_{\kappa^{*}+1}$ stand for $V$.

Claim 6.2. Given $\alpha<\beta$ in $N_{0} \cap(\kappa+1)$ and $G \in \mathrm{Gen}^{+}\left(N_{0}, P_{\alpha}, p\lceil\alpha) \cap N_{2 \alpha^{*}+1}\right.$, there is a $G^{\dagger} \in \operatorname{Gen}\left(N_{0}, P_{\beta}, p\right) \cap N_{2 \beta^{*}+1}$ such that whenever $r \in P_{\alpha}$ is a lower bound for $G$ that is $\left(N_{\xi}, P_{\alpha}\right)$-generic for $2 \alpha^{*}<\xi \leq \kappa^{*}$, there is an $r^{\dagger} \in P_{\beta}$ such that

(a) $r^{\dagger}$ is a lower bound for $G^{\dagger}$,

(b) $r^{\dagger} \uparrow \alpha=r$

(c) $r^{\dagger}$ is $\left(N_{\xi}, P_{\alpha}\right)$-generic for any $\xi$ with $2 \beta^{*}<\xi \leq \kappa^{*}$.

Proof of Claim 6.2. The proof is by induction on $\beta \in N_{0} \cap(\kappa+1) \backslash\{0\}$, for all $\alpha \in N_{0} \cap \beta$.

Case 1: $\alpha=0, \beta=1$. Recall that our convention is that $P_{0}$ is the trivial poset. The claim then reduces to the fact that $\dot{Q}_{0}$ is totally proper and $<\omega_{1}$-proper.

Case 2: $\beta=\beta_{0}+1$. Note that $\beta_{0} \in N_{0} \cap \beta$ as well. If $\alpha<\beta_{0}$, an application of the induction hypothesis with $\beta_{0}$ in place of $\beta$ gives us a $G_{0}^{+}$and $r_{0}^{+}$that we can 
use together with the following argument for the case $\alpha=\beta_{0}$. Thus we may as well assume that $\alpha=\beta_{0}$.

Inside the model $N_{2 \beta^{*}+1}$, we apply the definition of " $\dot{Q}_{\alpha}$ is 2-complete for $P_{\alpha}$ " with $N_{0}, N_{2 \alpha^{*}+1}, N_{2 \beta^{*}}, G$, and $p(\alpha)$ in place of the $N_{0}, N_{1}, N_{2}, \bar{G}$, and $\dot{q}$ there.

This gives us an $\bar{H} \in \operatorname{Gen}\left(N_{0}[G], \dot{Q}_{\alpha}, p(\alpha)\right) \cap N_{2 \beta^{*}+1}$ so that whenever $r$ is a lower bound for $G$ that is $\left(N_{\xi}, P_{\alpha}\right)$-generic for $\xi=2 \alpha^{*}+1,2 \beta^{*}$,

$$
r \Vdash \bar{H} \text { has a lower bound in } \dot{Q}_{\alpha} \text {. }
$$

Let $\dot{s} \in N_{2 \beta^{*}+1}$ be a $P_{\alpha}$-name forced by every such $r$ to be a lower bound for $\bar{H}$.

Define $G^{\dagger}$ as $\{u * \dot{v}: u \in G$ and $\dot{v} \in \bar{H}\}$. Standard arguments show that $G^{\dagger} \in \operatorname{Gen}\left(N_{0}, P_{\beta}, p\right)$, and $G^{\dagger} \in N_{2 \beta^{*}+1}$.

Now suppose $r$ is any lower bound for $G$ that is $\left(N_{\xi}, P_{\alpha}\right)$-generic for $2 \alpha^{*}<\xi \leq$ $\kappa^{*}$. Then $r$ forces that $\dot{s}$ is a lower bound for $\bar{H}$, and furthermore, there is $\dot{t}$ such that

$$
r \Vdash \dot{t} \leq \dot{s} \text { is }\left(N_{\xi}[\dot{G}], \dot{Q}_{\alpha}\right) \text {-generic for } 2 \beta^{*}<\xi \leq \kappa^{*},
$$

as $\Vdash_{\alpha} \dot{Q}_{\alpha}$ is $<\omega_{1}$-proper. We finish by defining $r^{\dagger}=r * \dot{t}$.

Case 3: $\beta$ a limit ordinal. Work for the moment in the model $N_{2 \beta^{*}+1}$. Fix $\left\{\alpha_{n}: n \in \omega\right\}$ strictly increasing and cofinal in $N_{0} \cap \beta$, with $\alpha_{0}=\alpha$. Let $\left\{D_{n}: n \in \omega\right\}$ list those dense open subsets of $P_{\beta}$ that are members of $N_{0}$.

We will define $\left\{p_{n}: n \in \omega\right\}$ and $\left\{G_{n}: n \in \omega\right\}$ that satisfy

1. $p_{0}=p, G_{0}=G$;

2. $p_{n+1} \in N_{0} \cap D_{n}$;

3. $p_{n+1} \leq p_{n}$

4. $p_{n} \uparrow \alpha_{n} \in G_{n}$;

5. $G_{n} \in \operatorname{Gen}\left(N_{0}, P_{\alpha_{n}}, p\left\lceil\alpha_{n}\right) \cap N_{2 \alpha_{n}^{*}+1}\right.$;

6. whenever $r \in P_{\alpha_{n}}$ is a lower bound for $G_{n}$ that is $\left(N_{\xi}, P_{\alpha_{n}}\right)$-generic for $2 \alpha_{n}^{*}<\xi \leq \kappa^{*}$, there is an $r^{\prime} \in P_{\alpha_{n+1}}$ such that

- $r^{\prime}$ is a lower bound for $G_{n+1}$,

- $r^{\prime}\left\lceil\alpha_{n}=r\right.$,

- $r^{\prime}$ is $\left(N_{\xi}, P_{\alpha_{n+1}}\right)$-generic for any $\xi$ with $2 \alpha_{n+1}^{*}<\xi \leq \kappa^{*}$.

Given $p_{n}$ and $G_{n}$, note that $E=\left\{t\left\lceil\alpha_{n}: t \in D_{n}, t \leq p_{n}\right\}\right.$ is dense below $p_{n}\left\lceil\alpha_{n}\right.$ and an element of $N_{0}$. Since $p_{n}\left\lceil\alpha_{n} \in G_{n}\right.$, we can find $t \in E \cap G_{n}$. Applying the definition of $E$ inside of $N_{0}$ gives us a $p_{n+1} \in N_{0} \cap D_{n}$ with $p_{n+1} \leq p_{n}$ and $p_{n+1}\left\lceil\alpha_{n} \in G_{n}\right.$.

The required $G_{n+1}$ is obtained by applying our main induction hypothesis with $\alpha_{n}, \alpha_{n+1}, p_{n+1}\left\lceil\alpha_{n+1}\right.$, and $G_{n}$ in place of $\alpha, \beta, p$, and $G$.

Once we have the sequences $\left\{p_{n}: n \in \omega\right\}$ and $\left\{G_{n}: n \in \omega\right\}$, define

$$
G^{\dagger}=\left\{t \in N_{0} \cap P_{\beta}: \exists n\left[t \geq p_{n}\right]\right\} .
$$

Our construction guarantees that $G^{\dagger} \in \operatorname{Gen}\left(N_{0}, P_{\beta}, p\right)$, and since we did the construction inside of $N_{2 \beta^{*}+1}$, we know $G^{\dagger}$ is in this model as well.

Subclaim 1. $G^{\dagger}$ has a lower bound.

To see this, let $r$ be a lower bound for $G$ that is $\left(N_{\xi}, P_{\alpha}\right)$-generic for $2 \alpha^{*}<\xi \leq$ $\kappa^{*}$. The properties of the sequence $\left\{G_{n}: n \in \omega\right\}$ allow us to build a sequence $\left\{r_{n}: n \in \omega\right\}$ satisfying

- $r_{0}=r$, 
- $r_{n}$ is a lower bound for $G_{n}$ in $P_{\alpha_{n}}$,

- $r_{n}$ is $\left(N_{\xi}, P_{\alpha_{n}}\right)$-generic for $2 \alpha_{n}^{*}<\xi \leq \kappa^{*}$,

- $r_{n+1} \uparrow \alpha_{n}=r_{n}$.

Finally, we let $r^{+}=\bigcup r_{n} \in P_{\beta}$.

Now why is $r^{+}$a lower bound for $G^{\dagger}$ ? Since $r_{n+1} \uparrow \alpha_{n}=r_{n}$ and $G_{n+1} \uparrow P_{\alpha_{n}}$ is a member of $\operatorname{Gen}\left(N_{0}, P_{\alpha_{n}}\right)$, by Lemma 3.6 we have that $G_{n+1} \uparrow P_{\alpha_{n}}=G_{n}$. From this, it follows easily by induction that $G_{m} \uparrow P_{\alpha_{n}}=G_{n}$ for $m>n$.

For $n \in \omega$, we know that $p_{n} \uparrow \alpha_{n} \in G_{n}$. If $m<n$, then $p_{m} \uparrow \alpha_{n} \geq p_{n} \uparrow \alpha_{n}$, so $p_{m} \uparrow \alpha_{n} \in G_{n}$. If $m>n$, then $p_{m}\left\lceil\alpha_{n} \in G_{m} \uparrow \alpha_{n}=G_{n}\right.$. Thus for each $n$, we have

$$
\left\{p_{m} \uparrow \alpha_{n}: m \in \omega\right\} \subseteq G_{n} .
$$

Since $r_{n}=r^{+} \uparrow \alpha_{n}$ is a lower bound for $G_{n}$, it follows that $r^{+}$is a lower bound for $G^{\dagger}$ and the subclaim holds.

To finish the proof of the claim for this case, let $r \in P_{\alpha}$ be a lower bound for $G$ that is $\left(N_{\xi}, P_{\alpha}\right)$-generic for $2 \alpha^{*}<\xi \leq \kappa^{*}$; we must produce a suitable $r^{\dagger}$.

Let $H \subseteq P_{\alpha}$ be generic with $r \in H$, and step into the extension $V[H]$. Recall that Lemma 3.6 implies $N_{0} \cap H=G$.

The proof of the subclaim tells us (in $V$ ) that there is a lower bound $r^{+}$for $G^{\dagger}$ with $r^{+} \uparrow \alpha=r$. Since $r \in H$, this means (in $V[H]$ ) that $G^{\dagger}$ has a lower bound in $P_{\beta} / H$ (recall Equation (3.1)), and by elementarity this must hold in the model $N_{2 \beta^{*}+1}[H]$ as well. Thus we can find $s \in P_{\beta} \cap N_{2 \beta^{*}+1}$ with $s \uparrow \alpha \in H$ that is a lower bound for $G^{\dagger}$. Since $\mathbb{P}$ is a countable support iteration of $<\omega_{1}$-proper notions of forcing, we can find a $t \leq s$ in $P_{\beta} / H$ that is $\left(N_{\xi}[H], P_{\beta} / H\right)$-generic for $2 \beta^{*}<\xi \leq \kappa^{*}$ and such that the support of $t$ is a subset of $N_{\kappa^{*}}[H] \cap O n$.

Back in $V$, we have a $P_{\alpha}$-name $\dot{t}$ such that $r$ forces

- $\dot{t} \in P_{\beta} / \dot{G}_{P_{\alpha}}$,

- $\dot{t}$ is a lower bound for $G^{\dagger}$,

- $\dot{t}$ is $\left(N_{\xi}\left[\dot{G}_{P_{\alpha}}\right], P_{\beta} / \dot{G}_{P_{\alpha}}\right)$-generic for $2 \beta^{*}<\xi \leq \kappa^{*}$,

- the support of $\dot{t}$ is a subset of $N_{\kappa^{*}}\left[\dot{G}_{P_{\alpha}}\right] \cap O n$.

Since $r$ is $\left(N_{\kappa^{*}}, P_{\alpha}\right)$-generic, the last clause implies $r$ forces that the support of $\dot{t}$ is a subset of $N_{\kappa^{*}} \cap O n$. Thus the condition $r * \dot{t} \in P_{\alpha} * P_{\beta} / \dot{G}_{P_{\alpha}}$ has a canonical translation into a condition $r^{\dagger} \in P_{\beta}$ with $r^{\dagger} \uparrow \alpha=r$. The needed properties of $r^{\dagger}$ then follow immediately from the corresponding properties of $r * \dot{t}$.

To finish the proof of Theorem 4, we apply the claim with $\alpha=0$ and $\beta=\kappa$. The $r$ and $G$ are trivially available, since $P_{0}$ is by convention the trivial 1-element poset. This gives us a $G^{\dagger} \in \operatorname{Gen}\left(N_{0}, P_{\kappa}, p\right)$ with lower bound $r^{\dagger}$, and we are done.

Armed with the previous theorem, we are now in position to build a model of $Z F C+C H$ in which there are no Ostaszewski spaces.

Proof of Theorem 1. Starting with a model of $C H$, construct a countable support iteration $\mathbb{P}=\left\langle P_{\alpha}, \dot{Q}_{\alpha}: \alpha<\omega_{2}\right\rangle$ so that for each $\alpha<\omega_{2}$,

$$
\Vdash_{\alpha} \dot{Q}=P_{\dot{X}} \text { for some Ostaszewski space } \dot{X} \text {. }
$$

(If at some stage there is no Ostaszewski space, we need iterate no farther.) Assuming that $C H$ holds, $P_{X}$ is $\omega_{1}$-centered - any two conditions with the same first component are compatible - and this is enough to ensure that $P_{\omega_{2}}$ has the $\omega_{2}$-c.c. (see Chapter VII of [5]). Since the topology on an Ostaszewski space has size $\omega_{1}$, a standard bookkeeping argument allows us to catch our tail and ensure there are 
no Ostaszewski spaces in $V\left[G_{\omega_{2}}\right]$. By Theorem 4, we know that $\mathrm{CH}$ continues to hold in $V\left[G_{\omega_{2}}\right]$, and so Theorem 1 is established.

\section{REFERENCES}

1. U. Abraham and S. Todorcevic, Partition properties of $\omega_{1}$ compatible with $C H$, Fund. Math. 152 (1997), no. 2, 165-181. MR 98b:03064

2. Alan Dow, More set theory for topologists, Top. and Appl. 64 (1995), 243-300. MR 97a:54005

3. M. Goldstern, Tools for your forcing construction, Set Theory of the Reals (H. Judah, ed.), Bar-Ilan, 1993, pp. 305-360. MR 94h:03102

4. A. Ostaszewski, On countably compact, perfectly normal spaces, J. London Math. Soc. 14 (1976), 505-516. MR 55:11210

5. Saharon Shelah, Proper forcing, Springer-Verlag, New York, 1982. MR 84h:03002

6. - Proper and improper forcing, Perspectives in Mathematical Logic, Springer, Berlin, 1998. MR 98m:03002

Institute of Mathematics, The Hebrew University, Jerusalem, Israel

Current address: Department of Mathematics, Ohio University, Athens, Ohio 45701

E-mail address: eisworth@math.huji.ac.il

Department of Mathematics, University of Kansas, Lawrence, Kansas 66045-0001

E-mail address: roitman@math.ukans.edu 